\title{
Delay Reducing Techniques during Handoff in Wireless LAN
}

\author{
Nirmal Kaur ${ }^{1}$, Dr. Supreet Kaur ${ }^{2}$ \\ ${ }^{1}$ PG Scholar, Punjabi University Regional Centre for IT and Management, Mohali 160062 (India) \\ ${ }^{2}$ Assistant Professor, Punjabi University Regional Centre for IT and Management, Mohali 160062 (India)
}

\begin{abstract}
WLANs play a vital role in $4^{\text {th }}$ Generation $(4 G)$ cellular system due to its adaptability and mobility. The most important issue in wireless communication networks to provide seamless handoff. Handoff delay make of serious problem as a lot of research has been done in last few years to reduce the handoff delay incurred in the different level of wireless communication. The whole delay time of a handoff process is isolated into authentication, probe and re association delay time. So this paper reviews the brief description about the distinct handoff techniques used in wireless networks and analyze their merits and demerits. Some future research ideas are also suggested here.
\end{abstract}

Keywords: WLAN, Handoff, Access Points, Handoff Delay, NLP, Neighbor Graph

\section{Introduction}

A Wireless Local Area Network (WLAN) is an extremely well known wireless access technology for short distances these days, its gaining popularity because of its good mobility, high transmission capacity and important flexibility. WLAN is utilized to connect two or more devices by utilizing some wireless distribution method like spread range, for the most part it gives an association through an access point to wide area network and provide the ability to users to move within a local coverage area by still connected to network[1].

The fundamental issue of wireless LANs is handoff delay, when the continuous association between mobile node and corresponding node from one point of APs is exchanged to another neighboring point of APs due to low sign quality, is known as handoff. Since APs have little coverage radius so WLAN network have hundreds or even a large numbers of APs. To have continuous connectivity the user's terminal needs to change its connection from one AP to another as it moves and this procedure expend a lot of time because it includes disconnection to the current AP and searching for another AP. This process has generally three main elements: prior-AP, Posterior-AP and Station. Prior-AP is the AP to which the station had the connectivity prior to handoff whereas the AP to which the station gets connectivity after the handoff process is posterior-AP [2].

\subsection{Types of Handoff}

There are two fundamental types of handoff: Soft handoff and hard handoff. In hard handoff, the station must break from ongoing connection with prior-AP before connecting to the posterior-AP, for this reason such handoff is also known as break-before-make. In hard handoff only one station is occupied at a time and it is used by TDMA and FDMA systems. On the other hand if the old connection is maintained until the new connection is established it is called Soft handoff and also known as make-before-break. Soft handoff used by CDMA system. It gives uninterrupted service and thus provides QoS [3].

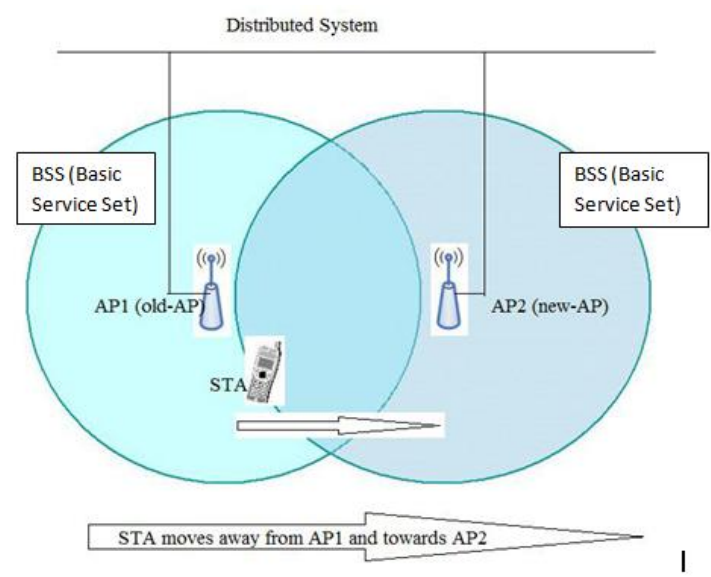

Figure 1: Handoff Architecture

Depend upon the transition in a wireless network; handoff can be classified into two parts: Horizontal handoff and Vertical handoff. Horizontal handoff is the transferring ongoing connection from one AP to another in different BSS and it is also called intra system handoff. whereas in vertical handoff ongoing connection is transferred between different wireless networks like wireless LAN to cellular and this type also called intersystem handoff [2].

\subsection{Handoff Performance metrics in WLAN}

Handoff is a key component in wireless networks since its conduct directly effects the general execution, regarding the QoS, signaling load and asset use [4]. A few measurements are utilized to access the performance of handoff techniques: for instance:

- Handoff probability: It indicates the probability that a handoff is happen when making a connection between nodes.

- Delay: It indicates the time interval between the starting of handoff demand and the execution of handoff demand. 


\section{International Journal of Science and Research (IJSR) \\ ISSN (Online): 2319-7064}

Index Copernicus Value (2013): 6.14 | Impact Factor (2015): 6.391

- Handoff blocking probability: it indicates the probability that a handoff endeavor is to be blocked.

- New call blocking probability: It is characterized as the probability that another endeavor call is to be blocked.

The rest of review paper is organized as follows. Section II briefly discussed about the handoff procedure. Section III illustrates the various handoff delay reducing techniques and section IV concludes the paper.

\section{Handoff Procedure}

During mobility when a station moves out from the extension scope of its previous AP and moves in another AP's area, it breaks the connection from the old AP and establishing the connection to new AP because of low signal quality [5]. This procedure of connection exchange is called handoff. The overall process of handoff has three phases: Authentication, scanning and re-association. Scanning is also known as probing. As shown in figure 2 Probing is the first step of Handoff procedure. In this step we select a reasonable AP from a neighboring AP to handoff. Scanning has two types: active and passive scanning. In active scanning, the station shows the "Probe Request" to all stations and waits for "Probe Response". It decreases the time taken to scan however builds the traffic loads. On the other side, in passive scanning each channel is recorded by station for the transmission of beacon frames. Passive scanning is mostly used in WLAN networks and it is power efficient.

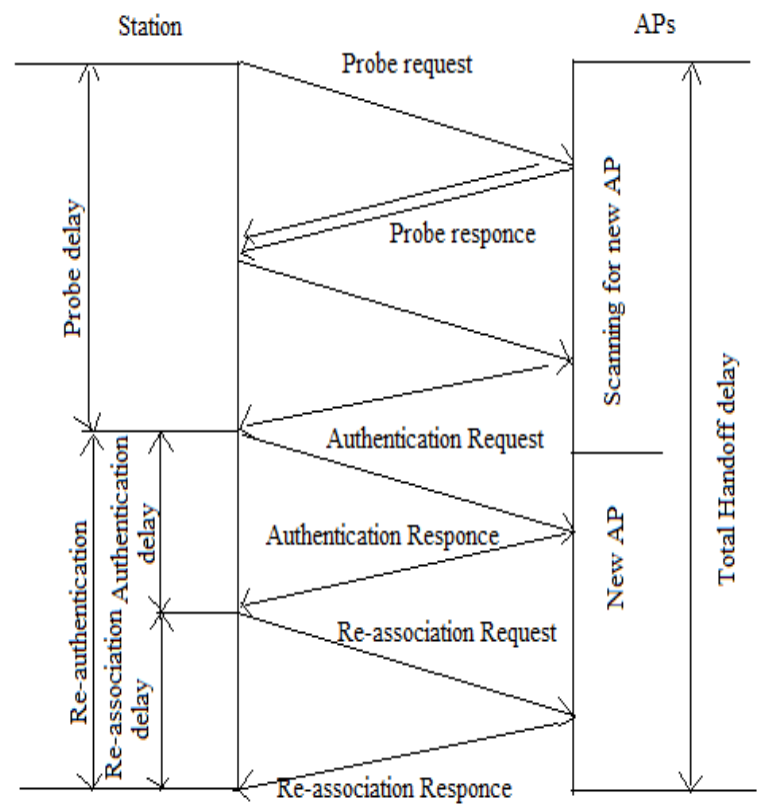

Figure 2: Handoff Process in WLAN

The next step of handoff process is to authenticate the new station [6]. For this, station sends authentication request to the selected AP and in response it send the authentication response. If the response is not received by station then it could not re-associate to the requested AP. The reassociation is to change the current association from an AP to another AP. The process of authentication and re-association is collectively known as re- authentication. The handoff delay is the sum of delay occurs in these phases.

\section{Various Handoff Delay Reducing Techniques}

There are a number of techniques used to reduce the handoff delay. A Review of some of these technologies is as given below:

\subsection{Handoff using Neighbor Graph (NG)}

The undirected Neighbor Graph (NG), whose every edge states a versatility path between APs and the neighbor of edge states the set of potential APs. The scheme proposed in [7] utilized the neighbor graph and sweep the channels taking into account position, speed and direction of the station using this technique and is used to get the data about neighbor AP. The station checks just those channels which are chosen by the neighbor graph. From the neighbor graph server, Station gets better scanned AP's data if there is necessity of handoff and handoff delay can be lessened. The overall execution of NG is as follow:

- After call setup the station screens the signal quality furthermore, measures relative signal strength (RSS) persistently.

- Station gets neighboring AP's sign at the point when it enters in the covered region.

- If current AP's $\mathrm{RSS}>=\mathrm{S}_{\mathrm{n}}$ the $\mathrm{NG}$ calculations begin and NG server save the data in cache. This procedure runs till $\mathrm{S}_{\mathrm{th}}=\mathrm{RSS}$.

- Station gets NG data and if current AP is available it sends signal message to that AP.

- The signal kernel table of current AP's is redesigns with SSID data of station.

- The station read the NG data once again, when old APs $\mathrm{RSS}=\mathrm{S}_{\mathrm{th}}$

- At the end the station chooses the best AP and send handoff start request. else it examines again using NG. 


\section{International Journal of Science and Research (IJSR) \\ ISSN (Online): 2319-7064}

Index Copernicus Value (2013): 6.14 | Impact Factor (2015): 6.391

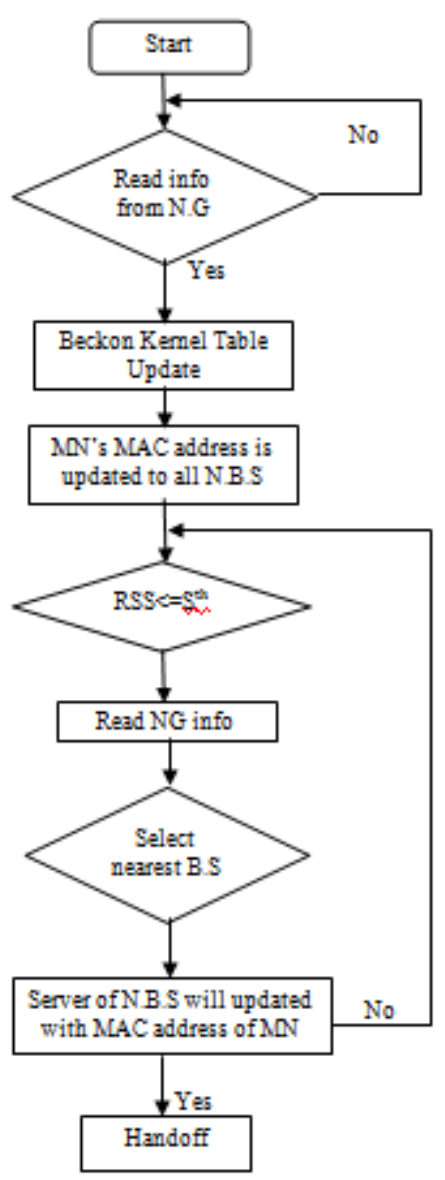

Figure 3: Handoff Execution of NG Client

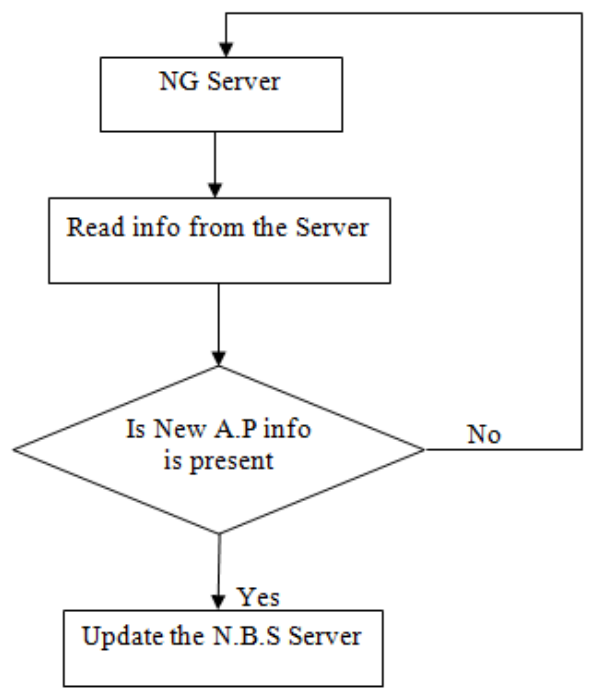

Figure 4: Handoff Execution of NG Server

The main advantages of this scheme are to sort out and manage the wireless systems and perform load balancing [8]. For fast handoff it eliminates the overpriced checking operation by constructing a wise speculation about the list of APs on specific channel.

\subsection{DeuceScan: Deuce- Based Fast Handoff scheme}

Further to reduce probe delay in WLAN, DeuceScan named technique is developed [9]. It utilizes a spatiotemporal graph which provides accurate information about space and time to reach to next APs. Based on that information an AP can make accurate Handoff decisions to next AP. Generally it is a pre-scan approach which efficiently reduces the MAC layer handoff delay. Signal strength and variation of signal strength, both factors considered in this technique. An important condition of this scheme is number of APs must be less than number of channels [10]. Advantage of the deuce process is to provide fault tolerant capability. If a handoff decision is made at time, we can make the correct decision from all accumulated results before time.

\subsection{HaND: Handoff with Null Dwell time Scheme}

This technique is depends on a novel zero-channel-dwelltime architecture means it does not require a station to dwell on the probed channels [11]. A station comes back to its present channel quickly after sending a probe frame on the new channel. This quality makes it different from existing handoff schemes. Rather, the current AP of the station is in charge of gathering the responses from nearby APs and sending to the station over its present channel. In the HaND, handoff choices are not made by the station itself but rather its currently associated AP. The overall communication process of HaND is shown in Fig.4.

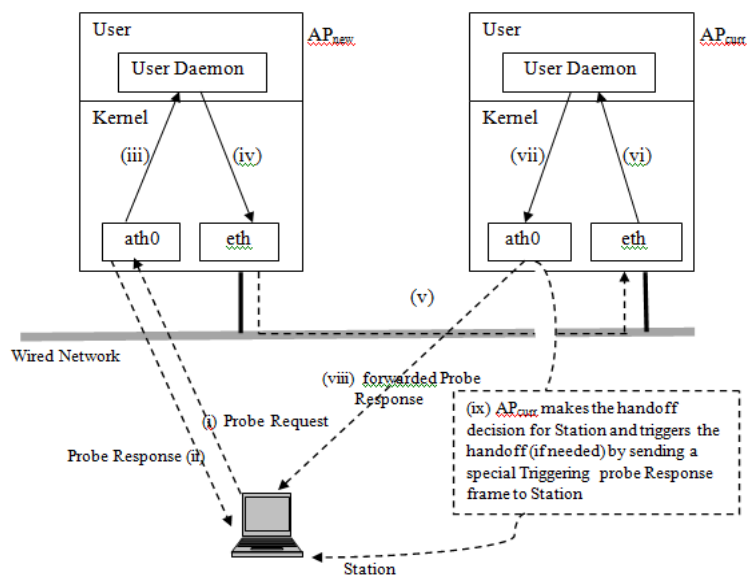

Figure 4: Overall structure of HaND scheme

- The station changes to channels CHnew and telecast a probe request edge, and after back changes to its working stations.

- After accepting the probe request frame from the station, AP new instantly answers a probe response frame through its wireless interface ath0.

- In expansion, APnew passes the data about its own channel, the RSSI estimation of the accepted Probe Request frame and a duplicate of produced Probe Response frame to user.

- The user frame at AP new thus sends this data to a user at APcurr through wired interface eth and wired communication backbone between APs.

- Upon getting the send data, APcurr redesign a nearby database that stores all the data forward from neighboring APs, produce a Forward Probe Response frame and telecast it through ath0.

So the main advantages of HaND are zero channel dwell time that makes it taking choices not only on the basis of station but its currently associated AP. 


\section{International Journal of Science and Research (IJSR) \\ ISSN (Online): 2319-7064}

Index Copernicus Value (2013): 6.14 | Impact Factor (2015): 6.391

\subsection{Pre-active scanning scheme}

The procedure of scanning is the most tedious; it is more than $90 \%$ of the aggregate handoff delay [12]. By utilizing the scheme of pre-active scanning, that is work with typical connective, it can reduce the aggregate of handoff delay during the procedure of detection and search stage. Mobile node can make pre-active scan direct immediately in period of detection and searching. The benefit of this scheme is that the mobile node can makes activity load sharing and takes decision to begin the handoff with the new AP channel which has a superior power signal than the most seasoned AP channel. Traffic load can increase in this scheme to save time for the telecast of a "probe request", and wait a "probe response" from neighbor AP channel. Likewise, Throughput is lessened, and traffic load is increased in the period of Preactive scan.

\subsection{Rotational Multiple Channel Allocation Scheme}

In this technique APs are responsible for portability of station to give quick handoff [13]. APs use $\mathrm{N}$ non-overlapping channels at the same time having $\mathrm{N}$ wireless interfaces. All APs and interfaces are set to the same medium access control (MAC) location and essential service set identity (BSSID). By setting the same BSSID, a MS accepts to coperate with the same AP by means of station moves to various AP. This scheme incorporates a Master AP (MAP) and various slave APs (SAPs). MAP is responsible to provide authentication, association and transmission duration (TD) for SAPs. The entire procedure for three cell cluster is as shown in Fig 5. There is no compelling reason to perform the ordinary handoff process. Performance evaluations demonstrate that this scheme can provide low handoff delay independent of the number of stations, payload sizes, and information rates.

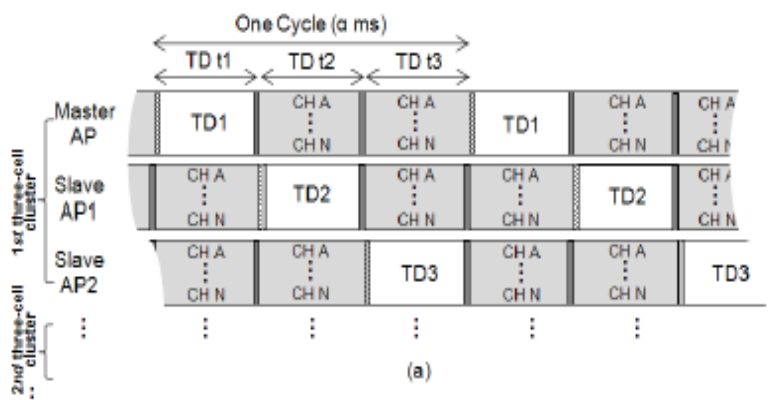

Figure 5: Rotational Multiple-Channel Handoff for Three Cell Cluster

\subsection{NLP based Handoff Scheme}

Neighbor list proactive (NLP) based handoff scheme use all the eleven channels to search a target AP based on RSSI [14]. One channel includes sending and receiving of probing packets over that channel. In this scheme controller is responsible for gathering APs data and circulating the neighbor list to APs. AP gets its continuous APs signal strength periodically and sends it to the controller and the controller creates a neighbor list or re-designs if it has already registered for each AP as shown in Fig.6. The greater part of the controllers can manage around 1024 APs (for instance, Cisco Access controller). The number of APs in the neighbor list can be set as consistent, additionally can be balanced in view of RSSI dynamically.

\section{Conclusion}

Wireless technologies are gaining popularity in each field, so there is a need to be always best connected and while roaming there should not be any delay in accessing services. To take full usage of wireless network, there should a minimum handoff delay. A number of techniques are used for this purpose. In this paper, we presented various handoff delay reducing techniques and their working procedures. We studied that FLAP is the best protocol as compared to other routing protocols so we can use it for fast handoff process.

\section{References}

[1] IEEE 802.11, "Information TechnologyTelecommunications and Information Exchange between Systems-Local and Metropolitan Area NetworksSpecific Requirements-Part 11: Wireless LAN Medium Access Control and Physical Layer Specifications," IEEE Standard, 2007.

[2] Pawar, Ms Madhuri R., and Santosh Sheshrao Sambare. "A Brief Review of Handover schemes in Wireless communication", vol. 3, no. 1, pp. 3269-3278,2012.

[3] Bhuvaneswari, A. "Survey On Handoff Techniques." Journal of Global Research in Computer Science 2, no. 6 (2011): 140-144.

[4] Martin-Escalona, Israel, Francisco Barcelo, and Jordi Casademont. "Teletraffic simulation of cellular networks: modeling the handoff arrivals and the handoff delay." In Personal, Indoor and Mobile Radio Communications, 2002. The 13th IEEE International Symposium on, vol. 5, pp. 2209-2213. IEEE, 2002.

[5] Mishra, Arunesh, Minho Shin, and William Arbaugh. "An empirical analysis of the IEEE 802.11 MAC layer handoff process." ACM SIGCOMM Computer Communication Review 33, no. 2 (2003): 93-102.

[6] Velayos, Hector, and Gunnar Karlsson. "Techniques to reduce the IEEE 802.11 b handoff time." In Communications, $2004 \quad$ IEEE International Conference on, vol. 7, pp. 3844-3848. IEEE, 2004.

[7] Sarddar, Debabrata, Tapas Jana, Tarasankar Patra, Utpal Biswas, and M. K. Naskar. "Fast handoff mechanism in WLANs based on neighbor graph information." In Parallel Distributed and Grid Computing (PDGC), 2010 1st International Conference on, pp. 334-338. IEEE, 2010

[8] Sarddar, Debabrata, Papri Mani, Utpal Biswas, and M. K. Naskar. "Fast Handoff Mechanism in Wireless Local Area Networks (WLAN) using Neighbor Graph Algorithm." International Journal of Computer Applications 25, no. 9 (2011): 36-40.

[9] Chen, Yuh-Shyan, Chung-Kai Chen, and Ming-Chin Chuang. "Deucescan: Deuce-based fast handoff scheme in ieee 802.11 wireless networks." InVehicular Technology Conference, 2006. VTC-2006 Fall. 2006 IEEE 64th, pp. 1-5. IEEE, 2006.

[10] Chen, Yuh-Shyan, Ming-Chin Chuang, and Chung-Kai Chen. "DeuceScan: deuce-based fast handoff scheme in 
IEEE 802.11 wireless networks." Vehicular Technology, IEEE Transactions on 57.2 (2008): 1126-1141.

[11] Chen, Xi, and Daji Qiao. "HaND: fast handoff with null dwell time for IEEE 802.11 networks." In INFOCOM, 2010 Proceedings IEEE, pp. 1-9. IEEE, 2010.

[12] Manodham, Thavisak, Mitsuo Hayasaka, and Tetsuya Miki. "A Novel Handover Scheme for Improving the Performance of WLANs based on IEEE802. 11." In Communications, 2006. APCC'06. Asia-Pacific Conference on, pp. 1-5. IEEE, 2006

[13]Jeon, Youchan, Myeongyu Kim, Sangwon Park, and Jinwoo Park. "Rotation multiple-channel allocation scheme for seamless handoff in IEEE 802.11 WLANs." In Consumer Electronics (ICCE), 2013 IEEE International Conference on, pp. 665-666. IEEE, 2013.

[14] Zhang, Hongchun, Zhaoming Lu, Xiangming Wen, and Zhiqun Hu. "QoE-Based Reduction of Handover Delay for Multimedia Application in IEEE 802.11 Networks." Communications Letters, IEEE 19, no. 11 (2015): 1873-1876.

\section{Author Profile}

Nirmal Kaur, Currently pursuing Master of Technology in Computer Science and Engineering from Punjabi University Regional Centre for IT and Management, Mohali (India). Before that she had done Bachelor of technology in Computer Engineering from YCOE, Campus of Punjabi University Patiala. 